\title{
A Video Recommendation System for Complex Topic Learning Based on a Sustainable Design Approach
}

\author{
Vargas Meza Xanat* \\ Department of Kansei, Behavioral and Brain Sciences \\ Graduate School of Comprehensive Human Sciences \\ The University of Tsukuba, 1-1-1 Tennodai Tsukuba \\ Ibaraki 305-8577, Japan \\ kt_designbox@yahoo.com \\ Yamanaka Toshimasa \\ Department of Art and Design, School of Art and Design \\ The University of Tsukuba, 1-1-1 Tennodai \\ Tsukuba, Ibaraki 305-8577, Japan \\ tyam@geijutsu.tsukuba.ac.jp \\ Received 30 December 2018 \\ Accepted 2 June 2019 \\ Published 8 July 2019
}

\begin{abstract}
There are several issues compromising the educational role of social networks, particularly in the case of video-based online content. Among them, individual (cognitive and emotional), social (privacy and ethics) and structural (algorithmic bias) challenges can be found. To cope with such issues, we propose a recommendation system for online video content, applying the principles of sustainable design. Precision and recall in English were slightly lower for the system in comparison to YouTube, but the variety of recommended items increased; while in Spanish, precision and recall were higher. Expected results include fostering the adoption of complex thinking by taking on account a user's objective and subjective contexts.
\end{abstract}

Keywords: Sustainable design; social networks; computational intelligence; information retrieval; multicriteria decision-making.

\section{Introduction}

The variety and availability of social networking sites in the Web have affected the way in which students learn. For example, some educators create Facebook groups, upload presentations in SlideShare or send assignments through Coursera. Those

*Corresponding author.

This is an Open Access article published by World Scientific Publishing Company. It is distributed under the terms of the Creative Commons Attribution 4.0 (CC-BY) License. Further distribution of this work is permitted, provided the original work is properly cited. 
who choose a self-learning approach can try websites like Khan Academy or videobased websites like TED. At the university level, it is thought that the adoption rate of social networking sites by faculty is over $90 \%,{ }^{32}$ with Facebook and YouTube being the most cited.

YouTube started as a self-expression and creativity focused video networking site in 2005, introducing advertisement one year later. The platform has been widely explored in relationship with education (see Refs. 8, 14, 18 and 22), with the most published studies noting its benefits. YouTube has also pioneered innovative contractual arrangements with corporations and users, ${ }^{3}$ although it is not exempted of issues.

Inappropriate content has been found targeting kids in YouTube. ${ }^{29}$ However, there are more subtle characteristics to take into account in terms of learning. Is the video too long? Is the video shot using objective or balanced remarks to explain a concept? Is the level of explanation fitting for the age or schooling of the user? Moreover, did the user have a bad day and is not in the mood to watch a maths or chemistry class? These are examples of cognitive and psychological aspects that not even the best data tracking and recommendation systems can predict and react to with accuracy in the case of open video data. Nevertheless, probably the most worrying trend is how YouTube creates addiction in adults and youngsters (see Refs. 7 and 30).

Having a hypothetical full access to track a user's behaviors, educational progress and emotions in a closed social networking environment would also imply issues. Current levels of data surveillance have brought undesired consequences such as leakage of personal information (e.g. Yahoo ${ }^{38}$ ), harassment and data-based discrimination. Moreover, the way companies collect and manage citizens' data in the Web is often not explained with clarity and several features cannot be opted out from. In the case of YouTube, unclear copyright terms for the content and a system that treats all matching videos as infringing content ${ }^{47}$ have led to muting and removal of videos, and even of user accounts. As can be noted in these examples, the delicate balance between an individual's rights and their usage of social networks is often at odds with other stakeholders' interests.

Finally, the structure of social networking sites has been shown to lead to echo chambers, particularly in the case of divisive topics (see Refs. 4 and 5). These studies argue that individual choices have a stronger effect than the algorithms, and there is evidence that people are exposed to diverse content but chose to embrace and amplify what is congruent with their ideologies. ${ }^{17}$ However, the impacts of advertising and propaganda posing as information contained in a video should also be taken in account. Recommendation algorithms have often been fooled to fuel harmful content, as political activities in the American continent and Spain recently showed (see Refs. 9 and 26).

The former two issues stem from the origins of computing, which have a direct relationship with public administration. According to Penn, ${ }^{36}$ French civil engineer de Prony developed logarithms based on Smith's The Wealth of Nations, which inspired Babbage's Difference Engine (subsidized by the government). Simon, winner 
of the Nobel Prize in economics for developing bounded rationality, translated this work into software code supported by America's Air Force in the mid 1950s. ${ }^{36}$ This was the first working prototype of Artificial Intelligence, which up to today mirrors bureaucratic institutions instead of originality, creativity or morals.

In sum, social networking sites monetize users' data with harmful consequences in the social sphere. Given that sustainability is the capacity to endure, embodied in long-term responsibility for the economic, social and ecological aspects of our world, and that the social dimension includes ensuring health, education, gender equality, inclusivity, peace and justice, ${ }^{49}$ social networking sites should ideally align with these principles. For all the aforementioned reasons, the objective of the present study is to explore alternative ways to manage YouTube's content through a recommendation system, enhancing it for educational purposes.

\section{State of the Art}

The expansion of the Web prompted the development of automated methods for information retrieval. A relevant aspect of recommender systems is the type of data input. Collaborative filtering systems employ user's ratings, content-based systems are more focused on the items' attributes and social- or knowledge-based systems manage data from more than one user at the same time. Hybrid approaches can employ two or more types of data. A review of recommender systems from the latter type can be found in Ref. 16. Also, Zhu et al. ${ }^{50}$ employed cross-curricular items.

Adomavicius and Tuzhilin ${ }^{2}$ described limitations of recommendation systems and discussed the possible areas of improvement, including users and items understanding, incorporation of contextual information, multicriteria ratings and more flexible and less intrusive recommendations. Among the possible methods to achieve improvement in recommendation systems, soft computing approaches have been widely researched. Such approaches can be classified into five types according to Yera and Martinez ${ }^{48}$ :

- Markov models: Stochastic processes that produce the sequence of observed symbols, ${ }^{39}$ based on complex probabilistic models.

- Bayesian approaches: Processes that "learn" patterns by using data from similar target users. They can also be based on statistical models, with Euclidean distance and naive Bayesian classifiers being frequently employed for recommendation systems. Lu et al. ${ }^{27}$ focused on recommendations' diversity based on surprise.

- Bio-inspired approaches: They mimic an aspect found in nature.

- Neural networks: These approaches "learn" patterns through previously collected data as well. The difference lies in the architecture of the networks, composed of aggregates of regularly spaced circuit clones (called cells), communicating with each other through their nearest neighbors. ${ }^{41} \mathrm{Ma}$ et al. $^{28}$ employed neural networks, user characteristics, keywords and keyframes for video recommendations. 
- Genetic algorithms: They are based on evolutionary principles such as natural selection and survival of the fittest, which are mainly used for data clustering and to aid user-based models. ${ }^{6}$

- Fuzzy logic: Processes that model real-world concepts which cannot be represented in precise ways, where elements have a degree of membership into a given set.

The YouTube recommendation algorithm is assumed to be based on neural networks, which model implicit feedback to provide fresh, time-consuming content at a large scale (see Refs. 11 and 26). However, human behavior and knowledge processes can be considered as fuzzy, as fuzzy logic represents the real world based on a degree of membership for elements into a given set. Fuzzy logic in recommender systems has been mostly focused on item profiling through tags and rates. A survey ${ }^{48}$ on over 100 algorithms found that many have not been tested with real-world data, and when they are, it has been on entertainment-related content. Leung et al. ${ }^{25}$ were the only ones who considered interest.

Regarding emotions, Trung et al. ${ }^{44}$ modeled fuzzy propagation for opinion mining, providing evidence of a relationship between specific sentiments and topics. Niu et $a l .{ }^{33}$ matched basic emotions with acoustic and visual features (such as color) of the video. However, none of the revised approaches combines diversity and emotion elicited by items, and also the level of interest, context and privacy of users in an educational scenario.

\section{Problem Definition}

\subsection{Learning a complex topic}

Although the terms "difficult" and "complex" overlap, a distinction between them should be made. We measure how difficult is a problem through the amount of effort that is required to solve it, while the solution is usually fixed, evaluated in terms of accuracy. Complexity, on the other hand, is measured in terms of depth of knowledge, understanding, awareness and strategy, which gives it a qualitative dimension. Complex problems can have multiple solutions or no solution at all.

Complex thinking presents a particular challenge in terms of education, as students have to deal with uncertainty. The latest proposals on pedagogy and research aim to re-link humans to the complex relations we have with other life forms and promote the learning of the "meaning of things for daily life" (see Refs. 15 and 37). Therefore, to understand complex topics, systems thinking and the ability to visualize relationships between elements are required.

\subsection{Sustainable design as a case of complex content}

Sustainable design takes into account the economic, social and ecological aspects in the whole product/service lifecycle. Therefore, it can be considered as an exemplary 
case of complexity. It is thought that one of the reasons why sustainable design has not been mainstreamed is that designers, mostly trained to work for industrialized companies, find it difficult to visualize, understand and deal with complexity. ${ }^{10}$ However, a study ${ }^{46}$ found that English speaking viewers' perception of sustainable design in YouTube involves a complex set of varied actors; videos where there was more interaction tended to show complex content applied to real-life problems, and even pilot projects involved details and system thinking in the case of English and Spanish videos. In sum, the results suggest an acceptance of complexity by the viewers.

Such acceptance might be aided by several factors, including topics related to design (its context) and emotions. We extracted the comments in English and Spanish made on top videos from the previous study, ${ }^{46}$ calculated the frequent keywords and words co-occurrence with ConText, ${ }^{12}$ performed a modularity test to find word clusters in Gephi and conducted sentiment analyses with SentiStrength ${ }^{42}$ and the Syuzhet package ${ }^{31}$ for R. SentiStrength was adapted to detect positive and negative polarities in the design context, ${ }^{45}$ and Syuzhet categories include disgust, fear and sadness, which might be related to rejection of sustainable design. Hence, multiple rank regression analysis was performed in SPSS to find out how much a word cluster could predict the sentiment.

According to literature on text analysis, relations between words and sentiments were expected to be small. ${ }^{24}$ Therefore, we considered an $R^{2}$ equal or higher than 0.15 to be significant. Results in Table 1 indicate that people- and money-related topics were better predictors for emotions than engineering and design topics

Table 1. Regression analysis for top word clusters and sentiments.

\begin{tabular}{|c|c|c|c|c|c|c|c|}
\hline \multicolumn{4}{|c|}{ English } & \multicolumn{4}{|c|}{ Spanish } \\
\hline Cluster name & $F$ & $R^{2}$ & Adj. $R^{2}$ & Cluster name & $F$ & $R^{2}$ & Adj. $R^{2}$ \\
\hline People & 459.389 & 0.228 & 0.228 & Jorge & 58.618 & 0.282 & 0.278 \\
\hline Money & 397.567 & 0.205 & 0.205 & System & 53.481 & 0.264 & 0.259 \\
\hline American & 397.567 & 0.204 & 0.203 & Bullfighting & 51.402 & 0.256 & 0.252 \\
\hline System & 372.830 & 0.194 & 0.193 & Economy & 31.952 & 0.177 & 0.171 \\
\hline Sci-Tech & 350.388 & 0.184 & 0.184 & Latinamericans & 30.211 & 0.169 & 0.163 \\
\hline Hollistic & 343.372 & 0.181 & 0.181 & Earth & 25.173 & 0.145 & 0.139 \\
\hline Ignorance & 332.252 & 0.176 & 0.176 & Chile-US & 20.020 & 0.118 & 0.113 \\
\hline Information & 327.320 & 0.174 & 0.174 & Housing & 13.716 & 0.084 & 0.078 \\
\hline She & 323.556 & 0.173 & 0.172 & Lucia & 12.245 & 0.076 & 0.070 \\
\hline James & 310.230 & 0.167 & 0.166 & Energy & 11.454 & 0.071 & 0.065 \\
\hline Jacque & 240.613 & 0.134 & 0.134 & House & 10.131 & 0.064 & 0.057 \\
\hline Energy & 225.672 & 0.126 & 0.126 & Mexico & 9.467 & 0.060 & 0.053 \\
\hline Design & 151.152 & 0.089 & 0.088 & Information & 7.649 & 0.049 & 0.042 \\
\hline $\mathrm{He}$ & 122.799 & 0.073 & 0.073 & Adobe & 6.575 & 0.042 & 0.036 \\
\hline
\end{tabular}

Note: Independent variables: Positive polarity, negative polarity, anger, anticipation, disgust, fear, joy, sadness and trust. Regression $=9, P<0.005$. English $N=13,976$, residual $=13,966$. Spanish $N=1,351$, residual $=1,341$. 
Table 2. Summary of barriers for sustainable design behavior.

\begin{tabular}{ll}
\hline English comments & Spanish comments \\
\hline Environmental: & Environmental: \\
— Natural disasters & - Nature viewed as dirty \\
Systemic: & Systemic: \\
- Money* & - Money* \\
- Politics/Government* & - Politics/Government* \\
- Religion & \\
— War & Psycho-social: \\
Psycho-social: & - Ignorance* \\
- Ignorance* & - Incompetence \\
- People* & - People* \\
- Racism & \\
- Resistance to change & Structural: \\
Structural: & - Conventional methods \\
- Ineffectiveness* & - Ineffectiveness* \\
— Infeasible & - Information (quality and quantity)* \\
- Information (quality and quantity)* & - Ugliness* \\
\hline Ugliness* & \\
Hierarchical: & \\
— Loss of autonomy & \\
\hline Loss of freedom &
\end{tabular}

(the last ones in the list), confirming the importance of the human dimension in sustainable design. An exception would be the Spanish Bullfighting cluster, which was a campaign against bullfighting pushed by grassroots associations.

A qualitative analysis on comments also confirmed the barriers for sustainable design behaviors associated with negative feelings. The summary of issues found through word frequency analysis, co-occurrence analysis and qualitative analysis can be consulted in Table 2. Coincidences between the two languages were marked with asterisks. Several features that are directly related to design (lack of enough information) and its context (the politic and economic systems) are considered as barriers that discourage a change of methods. However, they can also be considered as characteristics of complex thinking that are perceived as overwhelming and may cause either cognitive dissonance or rejection.

\section{Proposed Approach}

Taking into account that content about a complex topic can be overwhelming and that reactions of viewers depend on their level of interest and acceptance of it, and also that the management of YouTube's content should reflect more responsibility towards its users, strategies to design the recommendation system based on Ehn et al., ${ }^{13}$ Ito $^{21}$ and Acaraglou ${ }^{1}$ were considered. Sustainable design should not only include energy consumption and material recycling (video content and code in this case), but also the visualization of technology as extended, adaptive and regenerative intelligence that aids psychological restoration. 
The first step to achieve this objective was embedding YouTube content in a webpage that only shows thumbnails and titles, reducing distractions to aid the viewer's focus on the video. The application does not store user information permanently to enhance privacy and flexibility of recommendations. Because the system should also offer varied content to avoid echo-chambers, some degree of randomization in the items is preferred. As the videos are called through the YouTube API, they still receive views and are not manipulated in a way that infringes the social network policies. Regarding the content itself, nonthreatening nature, graphic representations of subjective concepts, role models and concrete design processes and products are included in the selected videos. All these elements counteract the barriers listed in Table 2 and aid psychological restoration.

Exposure to emotions varies according to the classification of users into interested, indecisive and uninterested in the topic (based on Refs. 19 and 34). Sadness is related to love, so interested users might be able to stand exposure to it. Anticipation-related comments tended to evaluate the pros and cons of sustainable design in a neutral way, so this emotion was assigned as the starting point for indecisive users. Previous literature also argues that urgent and concrete arguments are better for uninterested users, so fear was chosen as their starting point. Successive videos would then include more positive emotions for all types of viewers, but if their watching time is short, it would mean they are bored or annoyed. In that case, interested and indecisive users would be exposed to more negative emotions, while uninterested users would be exposed to more positive emotions.

\subsection{Data preprocessing}

Content of the video databases was classified according to language, uploading date, type of speaker, type of design shown and continents of countries of the YouTube channel and/or disclosed countries of speakers. Polarity (average of the sum of SentiStrength negative and positive scores) and average Syuzhet scores (anger, anticipation, disgust, fear, joy, sadness and trust) for each video were also stored in the databases. An emotionality score of comments was computed using the following formula:

$$
e=(\mathrm{np} *-1)+\mathrm{pp}-2,
$$

where $e$ stands for emotionality, np stands for negative polarity and pp stands for positive polarity. The average of emotionality was considered as a proxy for interest in the video, given that arousing content is more likely to provoke a reaction in the user. Maximum fuzzy similarity of topics found in video comments was also included in the databases. It was calculated with Excel Fuzzy Lookup add-on, which compares how similar a text is to groups of words (in this case, the groups found with the modularity test). The advantage of this method is that the video is classified into many overlapping topic groups, besides being quick and easy to compute. 


\subsection{Recommendation method}

The input for the system is occupation, expertise field, country, sentiment and interest. The output is a list of recommended videos for the average user with the same characteristics (except for sentiment). System processing is as follows:

(1) User. A potential viewer selects the desired language. System. This activates the corresponding database. Emotionality and polarity scores are scaled from $[0,4]$ to $[0,10]$ and polarity scores are scaled from $[-4,4]$ to $[-5,5]$.

(2) User. Inputs variables for occupation, expertise field, country, interest (emotionality) related to the topic and sentiment about the topic (polarity). System. It defines the maximum list size as 20. It classifies the user into interested (emotionality $[6,10]$, polarity $[-5,5]$ ), indecisive (emotionality $[0,5]$, polarity $[1,5]$ ) or uninterested (emotionality $[0,5]$, polarity $[-5,0]$ ). It also assigns the sequence start for Syuzhet scores (shown underlined in Table 3).

(3) System. Listing of videos is done according to the calculation of similarity based on Euclidean distance. It is also divided into two processes. The first video is selected according to Syuzhet scores sequence and the most recent uploading year. Other videos are selected based on the most recent uploading year, occupation/type of the speaker, expertise field/type of the design shown, country/continent and emotionality.

(4) User. They click a video from the list in the left-hand side of the first video. System. There are four ways to handle the user request, as shown in Table 4.

Table 3. Sequence of video sentiment scores according to user classification.

\begin{tabular}{lccccccc}
\hline User & Joy & Trust & Anticipation & Sadness & Disgust & Anger & Fear \\
\hline Interested & 1 & 2 & 3 & $\frac{4}{4}$ & 5 & 6 & 7 \\
Indecisive & 1 & 2 & $\frac{3}{3}$ & 4 & 5 & 6 & 7 \\
Uninterested & 1 & 2 & & 5 & 7 \\
\hline
\end{tabular}

Table 4. Handling of new video request.

\begin{tabular}{ll}
\hline $\begin{array}{l}\text { For English Interested_User and Indecisive_User, } \\
\text { the time is equal or less than } 10 \text { seconds } \\
\text { go forward with Syuzhet scores sequence, }\end{array}$ & $\begin{array}{l}\text { For Spanish Interested_User and Indecisive_User, } \\
\text { equal or less than } 7 \text { seconds } \\
\text { else go backward with Syuzhet scores sequence. }\end{array}$ \\
$\begin{array}{l}\text { For English Uninterested_User, } \\
\text { else go backward with Syuzhet scores sequence. }\end{array}$ \\
$\begin{array}{l}\text { For Spanish Uninterested_User, } \\
\text { jump time is equal or less than } 10 \text { seconds } \\
\text { else go backward with Syuzhet scores sequence. }\end{array}$ & $\begin{array}{l}\text { jump to Sadness and then to Trust, } \\
\text { else go backward with Syuzhet scores sequence. }\end{array}$
\end{tabular}

Hence, we considered that Spanish speakers tend to click faster than English speakers. When handling a new video request, listing is based on the most recent uploading year, occupation/type of the speaker, expertise field/type of the design shown, country/continent, Syuzhet scores sequence and emotionality. 
(5) User. They type keywords in the search bar. System. The recommendation method is the same as described above, adding the topic variable.

(6) User. To quit the program, the user exits the browser.

\subsection{Structure}

Figure 1 shows the recommendation process. After the user chooses a language, a data input interface appears. Their data is stored in a temporary database that communicates with other two databases (Country/Continent and Video Information). Then, a third interface appears with the video recommendations. If the user wants to search by keywords, the system will select videos again, using the additional Topics database.

\section{Testing Results}

Table 5 shows the search cases for YouTube compared to the recommendation system in English. We calculated the average precision and recall scores based on whether the items matched a student of architecture located in top countries related to sustainable design according to Ref. 46 . We only included the values for student and architecture in US because they were the same for other countries with the same language. Average scores in English were lower for the system, but the content from more continents was included among the recommended items, which was an intended effect to increase variety.

At first, the Spanish term sostenible was employed to search videos, but it was found that the term sustentable was frequently used to refer to sustainable design. Both words are translated as sustainable in English. Therefore, search queries using the two terms were compared (Table 6). Only values for student and architecture in Spain were included because they were the same for other countries with the same language. Overall, the average scores were higher for the system, with content from unclear locations being considerably less present among recommended items. Low scores for sostenible in YouTube reflect a decline in the popularity of the term from 2015 to 2018.

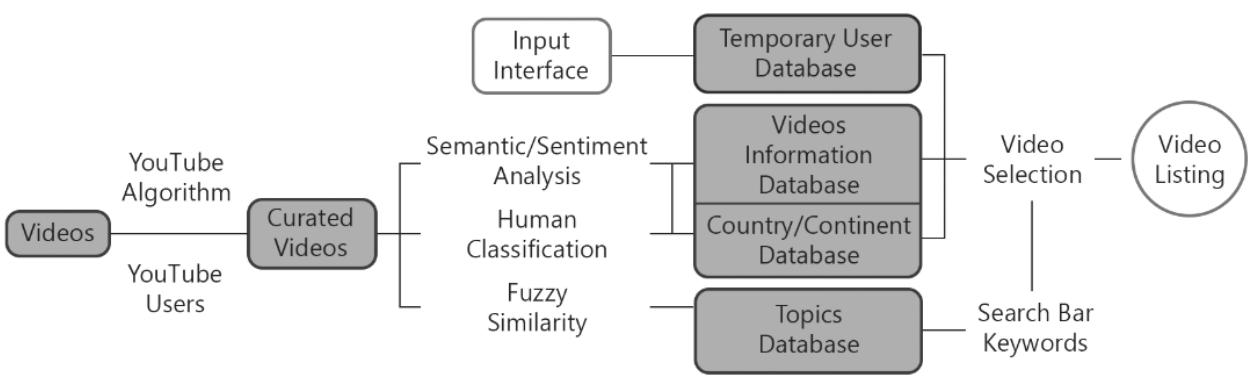

Fig. 1. Structure of the recommendation process. 
Table 5. Precision and recall scores for YouTube, and for recommendation system under interested, indecisive and uninterested settings in English videos.

\begin{tabular}{lcccccccc}
\hline & YT & YT & Sys Int & Sys Int & Sys Ind & Sys Ind & Sys Uni & Sys Uni \\
& Prec & Rec & Prec & Rec & Prec & Rec & Prec & Rec \\
\hline US Student & 0.075 & 0.075 & 0 & 0 & 0 & 0 & 0 & 0 \\
US Architecture & 0.375 & 0.375 & 0.450 & 0.450 & 0.375 & 0.375 & 0.375 & 0.375 \\
US N. America & 0.375 & 0.375 & 0.500 & 0.500 & 0.450 & 0.450 & 0.450 & 0.450 \\
US Average & 0.275 & 0.275 & 0.316 & 0.316 & 0.275 & 0.275 & 0.275 & 0.275 \\
UK Europe & 0.350 & 0.350 & 0.150 & 0.150 & 0.125 & 0.125 & 0.125 & 0.125 \\
UK Average & 0.266 & 0.266 & 0.200 & 0.200 & 0.166 & 0.166 & 0.166 & 0.166 \\
Australia Oceania & 0 & 0 & 0.100 & 0.100 & 0.050 & 0.050 & 0.050 & 0.050 \\
Australia Average & 0.150 & 0.150 & 0.150 & 0.150 & 0.158 & 0.158 & 0.158 & 0.158 \\
India S. Asia & 0 & 0 & 0.100 & 0.100 & 0.050 & 0.050 & 0.050 & 0.050 \\
India Average & 0.150 & 0.150 & 0.183 & 0.183 & 0.141 & 0.141 & 0.141 & 0.141 \\
Singapore E. Asia & 0 & 0 & 0.025 & 0.025 & 0.050 & 0.050 & 0.050 & 0.050 \\
Singapore Average & 0.150 & 0.150 & 0.158 & 0.158 & 0.141 & 0.141 & 0.141 & 0.141 \\
S. Africa Africa & 0 & 0 & 0.050 & 0.050 & 0.050 & 0.050 & 0.050 & 0.050 \\
S. Africa Average & 0.150 & 0.150 & 0.166 & 0.166 & 0.141 & 0.141 & 0.141 & 0.141 \\
\hline
\end{tabular}

Table 6. Precision and recall scores for YouTube, and for recommendation system under interested, indecisive and uninterested settings in Spanish videos.

\begin{tabular}{lcccccccccc}
\hline & & & & & Sys & Sys & Sys & Sys & Sys & Sys \\
& $\begin{array}{c}\text { YT } \\
\text { Prec }^{\mathrm{a}}\end{array}$ & $\begin{array}{c}\text { YT } \\
\text { Rec }^{\mathrm{a}}\end{array}$ & $\begin{array}{c}\text { YT } \\
\text { Prec }^{\mathrm{b}}\end{array}$ & $\begin{array}{c}\text { YT } \\
\text { Rec }^{\mathrm{b}}\end{array}$ & $\begin{array}{c}\text { Int } \\
\text { Prec }\end{array}$ & $\begin{array}{c}\text { Int } \\
\text { Rec }\end{array}$ & $\begin{array}{c}\text { Ind } \\
\text { Prec }\end{array}$ & $\begin{array}{c}\text { Ind } \\
\text { Rec }\end{array}$ & $\begin{array}{c}\text { Uni } \\
\text { Prec }\end{array}$ & $\begin{array}{c}\text { Uni } \\
\text { Rec }\end{array}$ \\
\hline Spain Student & 0.175 & 0.175 & 0.300 & 0.300 & 0.125 & 0.125 & 0.050 & 0.050 & 0.050 & 0.050 \\
Spain Architecture & 0.025 & 0.025 & 0.225 & 0.225 & 0.350 & 0.350 & 0.600 & 0.600 & 0.600 & 0.600 \\
Spain Europe & 0.266 & 0.266 & 0.025 & 0.025 & 0.375 & 0.375 & 0.150 & 0.150 & 0.150 & 0.150 \\
Spain Average & 0.155 & 0.155 & 0.183 & 0.183 & 0.283 & 0.283 & 0.266 & 0.266 & 0.266 & 0.266 \\
Colombia L. America & 0.341 & 0.341 & 0.650 & 0.650 & 0.550 & 0.550 & 0.650 & 0.650 & 0.650 & 0.650 \\
Colombia Average & 0.180 & 0.180 & 0.391 & 0.391 & 0.351 & 0.341 & 0.433 & 0.433 & 0.433 & 0.433 \\
\hline
\end{tabular}

${ }^{a}$ Keywords: diseño sostenible.

${ }^{\mathrm{b}}$ Keywords: diseño sustentable.

\section{Discussion}

Despite the distrust in social networking sites of public and governments in recent years, some scholars have highlighted their redeeming qualities. For example, Kampf $^{23}$ visualized YouTube as a multidimensional space for practice, user-to-user interaction, distributed problem-solving and dialog, with the potential to generate new knowledge.

In the case of the proposed recommendation system, interest in sustainable design would be either maintained in the case of advanced viewers or increased in the case of beginners, while concrete examples directly related to their expertise field and reactions by role models could motivate designers to realize the potential of sustainable design in their own practice and adopt it. Further, increasing the variety of recommended videos broadens the topics and "encompasses multiple ideologies and 
identities." ${ }^{20}$ Hence, the recommendation system acts as an automatic gatekeeper based on research by a human gatekeeper on videos chosen by yet another automatic gatekeeper (YouTube's algorithm) along with other human gatekeepers (those who interacted with the videos). In all these steps, human participation has been crucial.

It is tempting to develop a purely technological alternative to traditional education, diffused through the existing infrastructure with minimal cost and effort. However, a recommendation system paired with human guidance (e.g. a teacher) can address several issues simultaneously. An increasing dependence on modern technology fosters individualism, which is one of the reasons why designers fail to adopt sustainable design. ${ }^{43}$ Also, being exposed to only multimedia resources could harbor the experience of double reality, ${ }^{35}$ where information held in cognitive awareness cannot be integrated into daily life without compromising its integrity. This would reaffirm former views on the topic to be studied instead of contributing to change them.

The present results cannot claim to make lasting impacts on video viewers. What they reveal are the possibilities of conceptualizing technology in an educational context through sustainable design, while exposing viewers to the power dynamics between developed and developing countries. Answering to the question by Romero and Ventura ${ }^{40}$ on which affective states or student choices should we track besides interest and boredom when they interact with technology, the present study proposes to respect and embrace cultural differences in terms of context and emotional experience.

\section{Conclusions}

We proposed a video recommendation system for complex topic learning with a sustainable design approach in this paper. This is an application that can be downloaded and used anywhere with an Internet connection, does not store personal information and takes into account the users' feelings about a topic, advancing technology for the democratization and personalization of educative materials. Compared to YouTube, the proposed recommendation system offered more varied content to English speaking viewers, while Spanish speaking viewers would be exposed to more local content, in line with the average audience needs in both languages.

There are some limitations to our approach. Because of the wide range of analyses that were required to obtain the video databases, it is not possible to add more videos. Later versions could include a module to suggest videos, so that human experts can evaluate them before adding them to the databases. Automatizing the semantic, sentiment analyses and keywords classification would accelerate the process of adding videos to the databases. Another aspect that was out of this version's scope was videos in other languages, in order to cover the greater number of potential users in the world. Also, testing of the recommendation system with human subjects is highly recommended for future work. 


\section{Acknowledgments}

The authors wish to thank our reviewers for their contribution. This paper was supported by the Rotary Yoneyama Scholarship Foundation, as an extension of the Proposal of a Recommendation System for Complex Topic Learning Based on a Sustainable Design Approach, presented in the 10th International Conference on Computational Collective Intelligence (ICCCI 2018). We added comparisons with more countries in the English results and keywords in the Spanish results; enriched the literature review (Sec. 2), discussion (Sec. 6) and conclusions (Sec. 7); and clarified the recommendation method in text and figures.

\section{References}

1. L. Acaroglu, A manifesto for design-led systems change (2018), https://medium.com/ disruptive-design/a-manifesto-for-design-led-systems-change-28ac240db6dd.

2. G. Adomavicius and A. Tuzhilin, Toward the next generation of recommender systems: A survey of the state-of-the-art and possible extensions, IEEE Trans. Knowl. Data Eng. 17 (2005) 734-749.

3. M. Andrejevic, Exploiting YouTube: Contradictions of user-generated labor, in The YouTube Reader, eds. P. Snickars and P. Vonderau (The National Library of Sweden, 2009), pp. 406-423.

4. E. Bakshy, S. Messing and L. A. Adamic, Exposure to ideologically diverse news and opinion on Facebook, Science 348 (2015) 1130-1132, doi: 10.1126/science.aaa1160.

5. P. Barberá, J. T. Jost, J. Nagler, J. A. Tucker and R. Bonneau, Tweeting from left to right: Is online political communication more than an echo chamber? Psychol. Sci. 26 (2015) 1531-1542, doi: 10.1177/0956797615594620.

6. J. Bobadilla, F. Ortega, A. Hernando and A. Gutiérrez, Recommender systems survey, Knowl.-Based Syst. 46 (2013) 109-132.

7. B. Burroughs, YouTube kids: The app economy and mobile parenting, Soc. Media+Soc. 3 (2017) 2056305117707189, doi: 10.1177/2056305117707189.

8. S. C. Burke, S. L. Snyder and L. Shonna, Students' perceptions of YouTube usage in the college classroom, in Proc. Int. J. Instructional Technology and Distance Learning Vol. 5 (2008), pp. 13-23.

9. A. Cardenas, C. Ballesteros and R. Jara, Redes sociales y campañas electorales en Iberoamérica: Un análisis comparativo de los casos de España, México y Chile, Cuadernos. info 41 (2017) 19-40 (in Spanish).

10. C. M. Carter, Sustainable consumption \& sustainable design: Moving sustainability theory towards design practice, M.A. thesis (The University of Texas at Austin, USA, 2013).

11. P. Covington, J. Adams and E. Sargin, Deep neural networks for YouTube recommendations, in Proc. 10th ACM Conf. Recommender Systems (2016), pp. 191-198.

12. J. Diesner, ConText: Software for the integrated analysis of text data and network data, in Proc. Conf. Int. Communication Association (2014).

13. P. Ehn, I. Farías and T. S. Criado, On the possibility of socialist-democratic design things: Interview with Pelle Ehn, Diseña 12 (2018) 52-69.

14. C. J. Eick and D. T. J. King, Nonscience majors' perceptions on the use of YouTube video to support learning in an integrated science lecture, J. Coll. Sci. Teach. 42 (2012) 26-30.

15. M. Gadotti, Reorienting education practices toward sustainability, J. Educ. Sustain. Dev. 4 (2010) 203-211. 
16. L. Grad-Gyenge, A. Kiss and P. Filzmoser, Graph embedding based recommendation techniques on the knowledge graph, in Proc. Adjunct Publication of the 25th Conf. User Modeling, Adaptation and Personalization (ACM, New York, 2017), pp. 354-359.

17. M. Grossmann, Partisan Media and Political Distrust (Knight Foundation, USA, 2018).

18. J. J. Ham and M. A. Schnabel, Web 2.0 virtual design studio: Social networking as a facilitator of design education, Archit. Sci. Rev. 54 (2009) 108-116, doi: 10.1080/ 00038628.2011.582369.

19. D. W. Hine, W. J. Phillips, R. Cooksey, J. P. Reser, P. Nunn, A. D. Marks, N. M. Loi and S. E. Watt, Preaching to different choirs: How to motivate dismissive, uncommitted, and alarmed audiences to adapt to climate change? Glob. Environ. Change 36 (2016) 1-11, doi: $10.1016 /$ j.gloenvcha.2015.11.002.

20. D. Horton, Green distinctions: The performance of identity among environmental activists, Sociol. Rev. 51 (2003) 63-77.

21. J. Ito, Resisting reduction: A manifiesto - designing our complex future with machines (2017), https://pubpub.ito.com/pub/resisting-reduction.

22. A. Juhasz, Learning from YouTube (MIT Press, USA, 2011).

23. C. E. Kampf, Connecting corporate and consumer social responsibility through social media activism, Soc. Media+Soc. 4 (2018) 2056305117746357.

24. D. Kuster and A. Kappas, Measuring emotions in individuals and internet communities, in Internet and Emotions, eds. T. Benski and E. Fisher (Routledge, USA, 2013), pp. $48-64$.

25. C. W. K. Leung, S. C. F. Chan and F. L. Chung, A collaborative filtering framework based on fuzzy association rules and multiple-level similarity, Knowl. Inf. Syst. 10 (2006) 357-381, doi: 10.1007/s10115-006-0002-1.

26. P. Lewis, Fiction is outperforming reality: How YouTube's algorithm distorts truth (2018), https://www.theguardian.com/technology/2018/feb/02/how-youtubes-algorithmdistorts-truth.

27. W. Lu, F. L. Chung, W. Jiang, M. Ester and W. Liu, A deep Bayesian tensor-based system for video recommendation, ACM Trans. Inf. Syst. 37 (2018) 7.

28. J. Ma, G. Li, M. Zhong, X. Zhao, L. Zhu and X. Li, LGA: Latent genre aware micro-video recommendation on social media, Multimedia Tools Appl. 77 (2018) 2991-3008.

29. S. Maheshwari, On YouTube kids, startling videos slip past filters (2017), https://www. nytimes.com/2017/11/04/business/media/youtube-kids-paw-patrol.html.

30. K. Masters, Social networking addiction among health sciences students in Oman, Sultan Qaboos Univ. Med. J. 15 (2015) e357.

31. S. Mohammad and P. D. Turney, Crowdsourcing a word-emotion association lexicon, Comput. Intell. 29 (2013) 436-465, doi: 10.1111/j.1467-8640.2012.00460.x.

32. M. Moran, J. Seaman and H. Tinti-Kane, Teaching, Learning, and Sharing: How Today's Higher Education Faculty Use Social Media (Babson Survey Research Group, USA, 2011).

33. J. Niu, S. Wang, Y. Su and S. Guo, Temporal factor-aware video affective analysis and recommendation for cyber-based social media, IEEE Trans. Emerg. Top. Comput. 5 (2017) 412-424.

34. K. M. Norgaard, Living in Denial: Climate Change, Emotions, and Everyday Life (MIT Press, UK, 2011).

35. K. M. Norgaard, Normalizing the unthinkable: Climate denial and everyday life, in Twenty Lessons in Environmental Sociology, eds. K. A. Gould and T. Lewis (Oxford University Press, Oxford, 2014), pp. 246-259.

36. J. Penn, AI thinks like a corporation-and that's worrying (2018), https://www.economist.com/open-future/2018/11/26/ai-thinks-like-a-corporation-and-thats-worrying. 
37. C. Prado, in Forum Paulo Freire - V Encuentro Internacional: Sendas de Freire: Opresiones, Resistencias y Emancipaciones en un Nuevo Paradigma de Vida, eds. D. Monferrer, I. A. Guadas, P. M. Ortiz and P. A. Guadas (Instituto Paulo Freire de España y CREC, Xàtiva, 2006), pp. 169-211 (in Spanish).

38. M. Quick, E. Hollowood, C. Miles and D. Hampson, World's biggest data breaches: Information is beautiful (2018), http://www.informationisbeautiful.net/visualizations/ worlds-biggest-data-breaches-hacks.

39. L. Rabiner and B. Juang, An introduction to hidden Markov models, IEEE ASSP Mag. 3 (1986) 4-16.

40. C. Romero and S. Ventura, Data mining in education, Wiley Interdiscip. Rev., Data Min. Knowl. Discov. 3 (2013) 12-27.

41. R. J. Schalkoff, Artificial Neural Networks, Vol. 1 (McGraw-Hill, New York, 1997).

42. M. Thelwall, K. Buckley, G. Paltoglou, D. Cai and A. Kappas, Sentiment strength detection in short informal text, J. Assoc. Inf. Sci. Technol. 61 (2010) 2544-2558, doi: 10.1002/asi.21416.

43. C. Tonkinwise, The structure of structural change: Making a habit of being alienated as a designer, in Routledge Handbook of Sustainable Design, ed. R. B. Egenhoefer (Routledge, UK, 2018), pp. 433-445.

44. D. N. Trung, J. J. Jung, L. A. Vu and A. Kiss, Towards modeling fuzzy propagation for sentiment analysis in online social networks: A case study on TweetScope, in Proc. IEEE 4th Int. Conf. Cognitive Infocommunications (2013), pp. 331-338.

45. X. Vargas Meza and T. Yamanaka, Development of a sustainable design lexicon: Towards understanding the relationship between sentiments, attitudes and behaviours, in Proc. Int. Conf. Computational Collective Intelligence (2017), pp. 367-375.

46. X. Vargas Meza and T. Yamanaka, Sustainable design in YouTube: A comparison of contexts, Int. J. Affect. Eng. 17 (2017) 39-48, doi: 10.5057/ijae.IJAE-D-17-00010.

47. F. von Lohmann, YouTube's January fair use massacre (2009), https://www.eff.org/ deeplinks/2009/01/youtubes-january-fair-use-massacre.

48. R. Yera, and L. Martinez, Fuzzy tools in recommender systems: A survey, Int. J. Comput. Intell. Syst. 10 (2017) 776-803, doi: 10.2991/ijcis.2017.10.1.52.

49. United Nations Department of Economic and Social Affairs (UNDESA), SDGS \& topics (2019), Sustainable Development Goals Knowledge Platform, https://sustainabledevelopment.un.org/topics.

50. H. Zhu, Y. Liu, F. Tian, Y. Ni, K. Wu, Y. Chen and Q. Zheng, A cross-curriculum video recommendation algorithm based on a video-associated knowledge map, IEEE Access 6 (2018) 57562-57571. 